Document downloaded from:

http://hdl.handle.net/10251/178930

This paper must be cited as:

Andrés, D.; Jiménez-Gambín, S.; Jimenez, N.; Camarena Femenia, F. (2020). Multifocal acoustic holograms for deep-brain neuromodulation and BBB opening. IEEE. 1-3. https://doi.org/10.1109/IUS46767.2020.9251769

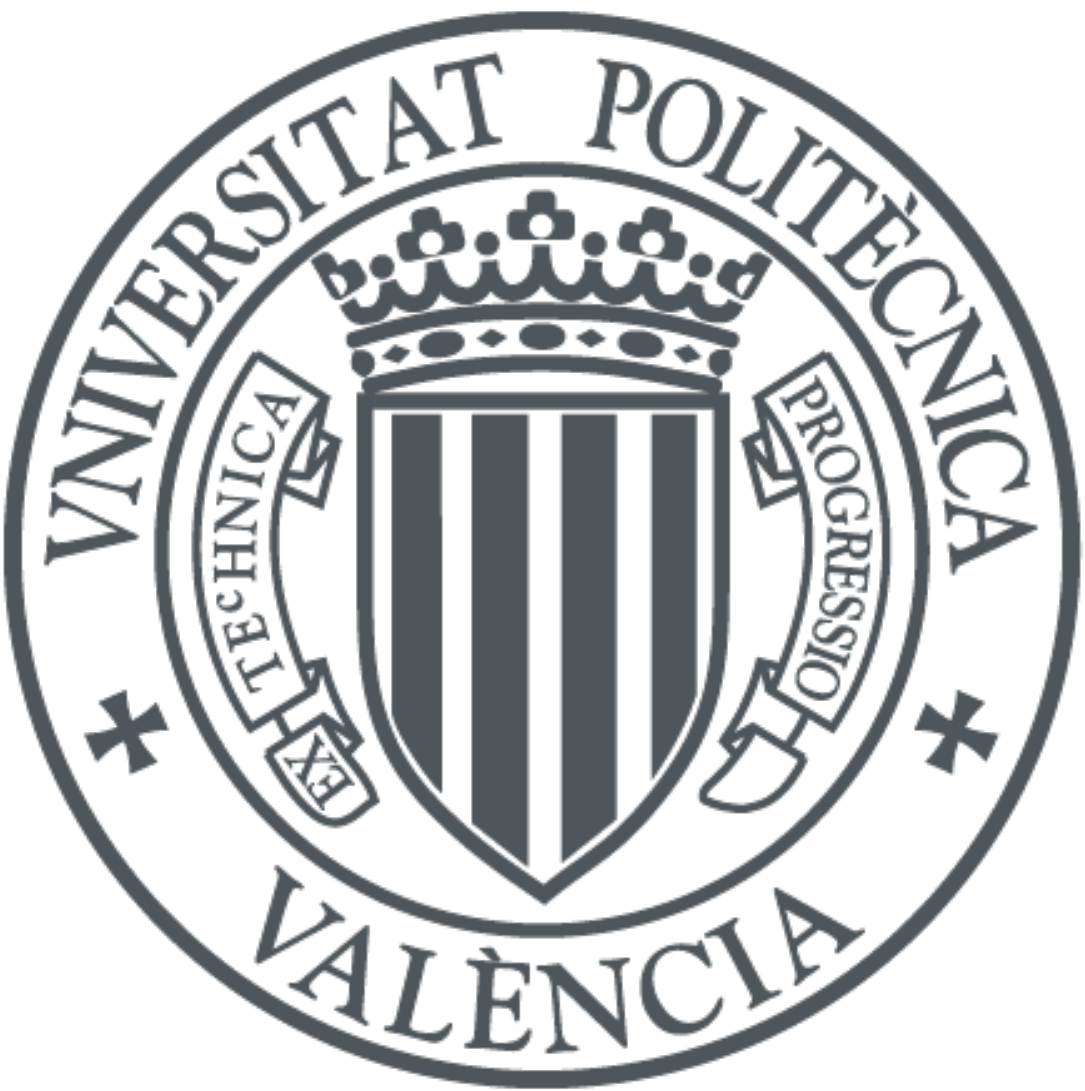

The final publication is available at

https://doi.org/10.1109/IUS46767.2020.9251769

Copyright IEEE

Additional Information 


\title{
Multifocal acoustic holograms for deep-brain neuromodulation and BBB opening
}

\author{
Diana Andrés*, Sergio Jiménez-Gambín, Noé Jiménez and Francisco Camarena \\ Instituto de Instrumentación para Imagen Molecular \\ Universitat Politècnica de València - CSIC, València, Spain \\ *diaanbau@upv.es
}

\begin{abstract}
Single-element focused ultrasound devices have demonstrated its ability to non-invasively open the Blood Brain Barrier. However, skull irregularities and absorption produce strong aberrations in the ultrasound focus. Recently, 3D-printed acoustic holograms were used to compensate aberrations and to produce a sharp focus. In addition, using this technology the geometry of the ultrasonic focus can be matched to the shape of the brain structure of interest. In this work, we experimentally and numerically report 3D-printed acoustic holograms for bilateral focusing through an ex-vivo human skull. Using holograms, we target different cerebral nuclei and the ultrasonic focuses are adapted to the target volumes, minimizing the acoustic field outside. Simultaneously, skull aberrations are corrected using phased-conjugation methods. The holographic surfaces were manufactured for a $100-\mathrm{mm}$ aperture and $140-\mathrm{mm}$ focal focused transducer at $500 \mathrm{kHz}$ to focus through an ex-vivo human skull. Numerical and experimental results agree targeting several deep-brain nuclei: left-and-right putamen, caudate nuclei, and hippocampi, showing the potential of this low-cost technology to optimize BBBO or neuromodulation treatments.
\end{abstract}

Index Terms-Acoustic holograms, therapeutic ultrasound, neuromodulation, blood-brain barrier opening.

\section{INTRODUCTION}

Focused ultrasounds have become a key therapeutic modality for the non-invasive and non-ionizing treatment of neurological disorders, which currently affect one billion people worldwide. Through thermal ablation of thalamic and subthalamic areas [1], treatments have been developed for essential tremor, Parkinson's disease or chronic pain [2], while at moderate intensity they are used for neuromodulation [3] or bloodbrain barrier opening to deliver drugs in a safe and localized manner [4]. However, focusing therapeutic ultrasound into the central nervous system has been limited by two major drawbacks: the aberrant and attenuating effects of the skull and the large mismatch between the volume of the brain structure to be treated and the volume of the ultrasonic beam focus, which in most cases forces multiple sonications to cover the desired area. In addition, current phased-array technology does not allow the simultaneous sonication of two or more spatially separated volumes. Recently, single-focus 3D-printed lenses

This research has been supported by the Spanish Ministry of Science, Innovation and Universities through grant "Juan de la Cierva - Incorporación" (IJC2018-037897-I) and PID2019-111436RBC22, by the Agencia Valenciana de la Innovació through grants INNVAL10/19/016 and INNCON/2020/009. Action cofinanced by the European Union through the Programa Operativo del Fondo Europeo de Desarrollo Regional (FEDER) of the Comunitat Valenciana 2014-2020 (IDIFEDER/2018/022).
[5] and acoustic holograms [6] were proposed to compensate the aberrations of the skull. In this work, we present the ex-vivo validation on a human skull of 3D-printed acoustic holograms to correct the aberrations introduced by the skull and, simultaneously, generate multifocal ultrasound beams which match the target structures in different parts of the human brain.

\section{MATERIALS AND METHODS}

To generate holograms inside the skull, we first use CT imaging to extract the hard-tissue shape and properties, and, second, MRI to identify the geometry and location of the target inside the brain, e.g., the caudate nuclei or both hippocampi. The information of phase and amplitude of the wavefront coming from the target is needed to generate the physical lens, so virtual sources are set at the structure location and a numerical back-propagation method is used. Then, the lens will be designed using this information and will match the curvature of the focal transducer. It is printed using 3D stereolithography and, then, hydrophone measurements in water tank are performed.

\section{A. Skull properties and target segmentation}

The ex-vivo skull acoustic properties have been obtained from a CT image (Philips Brilliance CT 64 at the Quirón Salud hospital, Valencia), with a resolution of $0.33 \times 0.33 \times$ $1.25 \mathrm{~mm}^{3}$. Conversion from Hounsfield Units (HU) to acoustic impedance have been done with existing empirical lineal fits for soft- an hard tissues [7] to experimental data [8]. The value of skull attenuation has been obtained from the literature [9] for $500 \mathrm{kHz}$ and it is considered as constant in all the skull, with a value of $5.08 \mathrm{~dB} /\left(\mathrm{cm} \cdot \mathrm{MHz}^{y}\right)$. Mean values of density and sound speed of the skull tissue are $1466 \mathrm{~kg} / \mathrm{m}^{3}$ and 2114 $\mathrm{m} / \mathrm{s}$, respectively.

The therapeutic targets considered in this study are the putamen and caudate nuclei and left- and right hippocampi. To obtain their shape, volume and location inside the brain we have considered data from the open atlas of the International Consortium for Brain Mapping (ICBM) from the Laboratory of Neuro Imaging (https://identifiers.org/neurovault.image:1401). This atlas consists of a MRI with color-coded slice segmentation of several cerebral nuclei. Volume segmentation for the target nuclei and brain tissue was performed using the ITKSnap software [10]. 


\section{B. Time reversal method}

To obtain the acoustic hologram we make use of the timereversal invariance of the acoustic propagation [11]. To directly use this method experimentally, it is necessary that a physical acoustic source is set inside the brain at the target location, which may require of a surgical intervention. However, backpropagated field can also be obtained by numerical simulations by setting a series of virtual sources at the location of the therapeutic targets [12]. Analogously to the physical acoustic source inside the brain, the virtual sources generate sound waves whose phase and amplitude can be captured to design a physical acoustic hologram. In this work, virtual sources are located at a plane perpendicular to the transducer, following the shape and size of the deep-brain nuclei. Numerical simulations were performed with the $k$-Wave MATLAB toolbox [13] which implements a $k$-space pseudo-spectral method for modelling the ultrasound propagation in heterogeneous media.

\section{Lens design}

To design the holographic lens, we use the complexconjugated pressure distribution recorded at the surface of the transducer. In this work, the holographic surface has the shape of the focused transducer curvature. The lens surface is divided into pixels in spherical coordinates, of uniform width, each one having different height, $r(\theta, \phi)$. Each pixel of the resulting lens is designed to be perpendicular to the curved transducer surface at each point. We assume that every truncated-pyramidal pixel vibrates longitudinally as an elastic Fabry-Perot resonator. Then, the phase of the transmitted waves can be fitted to the phase of the complex-conjugated recorded field by tuning the height of each pixel, as shown in Fig. 1, following the procedure described in Ref. [6].

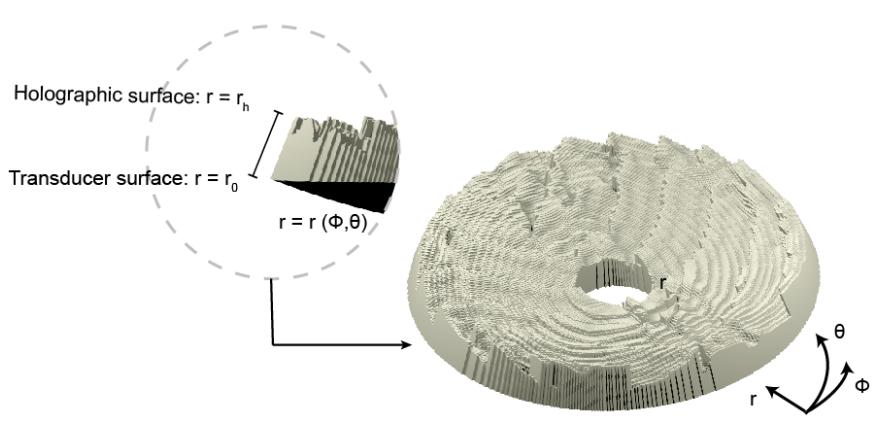

Fig. 1. Geometry of the holographic curved lens. The lens is subdivided in truncated-pyramidal pixels of height $r(\theta, \phi)$. The transducer is located at $r=r_{0}$ and the holographic surface at $r=r_{h}$.

The lens is manufactured using 3D stereolithography (SLA) techniques with a Form 2 printer (Formlabs, USA), with a resolution of $50 \mu \mathrm{m}$ and using the Clear photosensitive resin. The acoustical properties of the lens material were obtained experimentally, resulting in a measured sound speed of $c_{L}=2580 \mathrm{~m} / \mathrm{s}$ and a density of $\rho_{L}=1171 \mathrm{~kg} / \mathrm{m}^{3}$, and the absorption was set to $\alpha=1.38 \mathrm{~dB} / \mathrm{cm}$ at $500 \mathrm{kHz}$, matching the reported values of similar polymers [14].

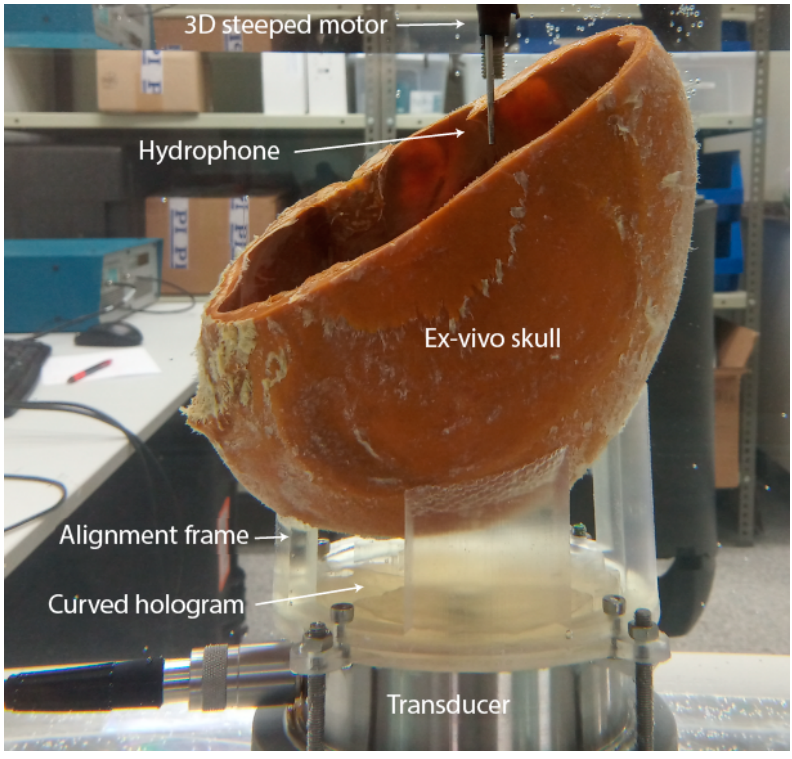

Fig. 2. Experimental setup for the acoustic field measurement.

\section{Experimental setup}

The experiments were conducted in a water tank at $24^{\circ} \mathrm{C}$ of $80 \times 43 \times 63 \mathrm{~cm}^{3}$. The ultrasonic transducer is composed of a curved piezoceramic with an aperture of $2 a=100 \mathrm{~mm}$ and a focal distance of $F=140 \mathrm{~mm}$. The piezoceramic is mounted in a custom designed stainless-steel housing. To align and fix the holographic lens to the skull a 3D printed holding support was 3D-printed with a shape taken from surface of the skull. The experimental setup is shown in Fig. 2. The transducer was driven with a 20 cycles sinusoidal pulse burst at a frequency of $f=500 \mathrm{kHz}$ by a signal generator (14 bits, $100 \mathrm{MS} / \mathrm{s}$, model PXI5412, National Instruments) and amplified by a linear RF amplifier (ENI 1040L, 400 W, 55 dB, ENI, Rochester, NY). The pressure field was measured by a needle hydrophone (-228 $\mathrm{dB}$ re $1 \mathrm{~V} / \mu \mathrm{Pa}$ at $500 \mathrm{kHz}$, Model TC4038, Teledyne RESON) calibrated from $10 \mathrm{kHz}$ to $800 \mathrm{kHz}$.

\section{RESULTS}

First, the acoustic field for three different bilateral therapeutic targets inside the brain was simulated. For the caudate nuclei, the volume covered in one sonication was 3.9 $\mathrm{mm}^{3}$, which represents $24 \%$ of the total target volume. (The sonicated volume is defined as the tissue sonicated with an intensity higher than half the peak intensity). Similar values are obtained for the putamen, where $25 \%$ of its volume is covered, and the hippocampus, with $22 \%$. The gain was $3.7 p / p_{0}, 3.5$ $p / p_{0}$ and $3.3 p / p_{0}$ for the caudate, putamen and hippocampus, respectively, where $\mathrm{p} 0$ is the pressure at the transducer surface.

In Fig. 3a we show the simulation using the acoustic hologram to focus on the hippocampus. Figures $3 b, 3 c, 3 d$ and $3 \mathrm{e}$ show the acoustic field measured experimentally and obtained by simulation. The 3D printed lens generates a pair of diffraction-limited focal spots matching the location of both virtual sources. Figure $3 \mathrm{f}$ compares both numerical 

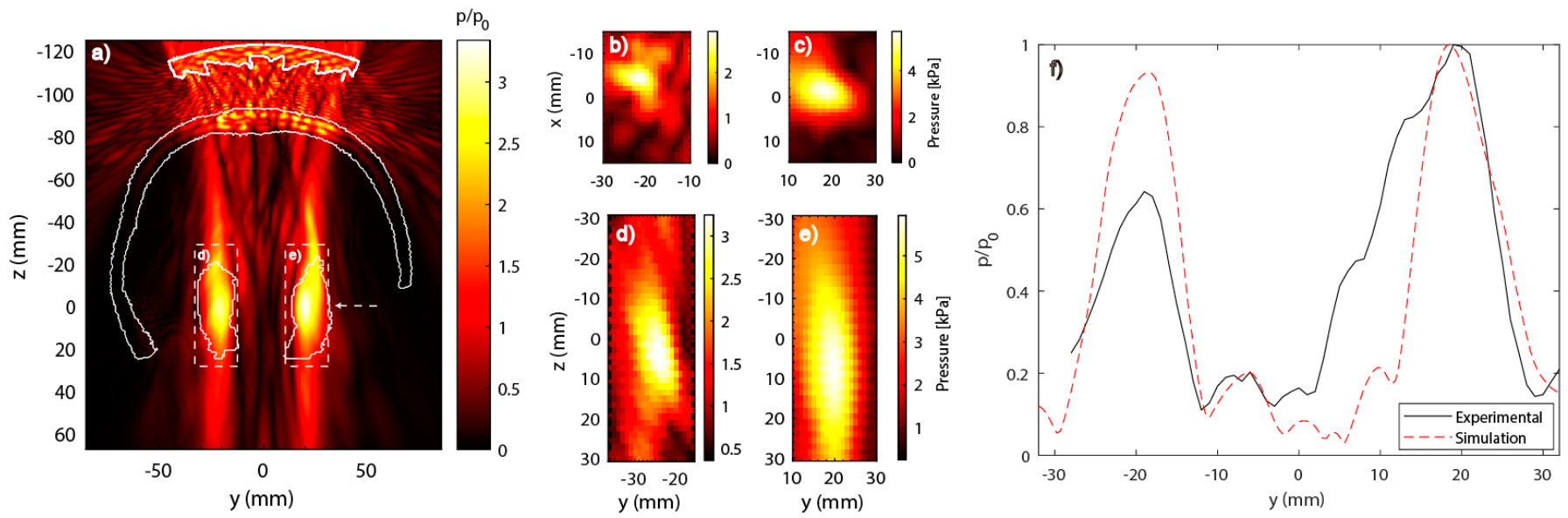

Fig. 3. Acoustic field matching both hippocampi. a) Simulation results: in the image are drawn the lens coupled to the curved transducer, the skull and the virtual sources cut following the hippocampus shape. The discontinuous line delimits the region where experimental measurements were done. Experimental results for b) a coronal cut in the plane $z=0$ following the shape of the left hippocampus and c) the right hippocampus, d) axial cut at $x=0$ following the shape of the left hippocampus and e) the right hippocampus. f) Experimental and simulation normalized pressure in a cut at $z=0$ and $x=-4$.

and experimental results, showing a good agreement between them. It is shown that the acoustic energy is mainly located at the targeting regions, allowing beam steering and bilateral focusing.

\section{CONCLUSIONS}

We have experimentally demonstrated that the shape of the acoustic field can be adapted to complex therapeutic targets using acoustic holograms coupled to single-element ultrasonic transducers in a simple and robust manner. In this way, acoustic holograms can compensate the aberrations of the wavefront produced by the skull while, simultaneously, they allow bilateral sonications whose acoustic field fits to deep brain structures such hippocampi, putamen, or caudate nuclei. In this work, numerical and experimental results focusing through an ex-vivo human skull show that more than $20 \%$ of the volume of bilateral targets inside the brain can be covered with a single configuration. Furthermore, the steering capabilities of the acoustic holograms have been demonstrated by focusing at three different structures using one single transducer-skull position. In this way, acoustic holograms might be used to design low-cost and robust systems to focus at complex targets for ultrasound neuromodulation and bloodbrain barrier opening applications.

\section{REFERENCES}

[1] F. Prada, M. Y. S. Kalani, K. Yagmurlu, P. Norat, M. Del Bene, F. DiMeco, and N. F. Kassell, "Applications of focused ultrasound in cerebrovascular diseases and brain tumors," Neurotherapeutics, vol. 16, no. 1, pp. 67-87, 2019.

[2] W. J. Elias, N. Lipsman, W. G. Ondo, P. Ghanouni, Y. G. Kim, W. Lee, M. Schwartz, K. Hynynen, A. M. Lozano, B. B. Shah et al., "A randomized trial of focused ultrasound thalamotomy for essential tremor," New England Journal of Medicine, vol. 375, no. 8, pp. 730739, 2016.

[3] W. Legon, T. F. Sato, A. Opitz, J. Mueller, A. Barbour, A. Williams, and W. J. Tyler, "Transcranial focused ultrasound modulates the activity of primary somatosensory cortex in humans," Nature neuroscience, vol. 17, no. 2, pp. 322-329, 2014
[4] N. Lipsman, Y. Meng, A. J. Bethune, Y. Huang, B. Lam, M. Masellis, N. Herrmann, C. Heyn, I. Aubert, A. Boutet et al., "Blood-brain barrier opening in alzheimer's disease using mr-guided focused ultrasound," Nature communications, vol. 9, no. 1, pp. 1-8, 2018.

[5] G. Maimbourg, A. Houdouin, T. Deffieux, M. Tanter, and J.-F. Aubry, "3d-printed adaptive acoustic lens as a disruptive technology for transcranial ultrasound therapy using single-element transducers," Physics in Medicine \& Biology, vol. 63, no. 2, p. 025026, 2018.

[6] S. Jiménez-Gambín, N. Jiménez, J. M. Benlloch, and F. Camarena, "Holograms to focus arbitrary ultrasonic fields through the skull," Physical Review Applied, vol. 12, no. 1, p. 014016, 2019.

[7] T. D. Mast, "Empirical relationships between acoustic parameters in human soft tissues," Acoustics Research Letters Online, vol. 1, no. 2, pp. 37-42, 2000.

[8] U. Schneider, E. Pedroni, and A. Lomax, "The calibration of ct hounsfield units for radiotherapy treatment planning," Physics in Medicine \& Biology, vol. 41, no. 1, p. 111, 1996.

[9] R. S. Cobbold, Foundations of biomedical ultrasound. Oxford university press, 2006.

[10] P. A. Yushkevich, J. Piven, H. C. Hazlett, R. G. Smith, S. Ho, J. C. Gee, and G. Gerig, "User-guided 3d active contour segmentation of anatomical structures: significantly improved efficiency and reliability," Neuroimage, vol. 31, no. 3, pp. 1116-1128, 2006.

[11] J.-L. Thomas and M. A. Fink, "Ultrasonic beam focusing through tissue inhomogeneities with a time reversal mirror: application to transskull therapy," IEEE transactions on ultrasonics, ferroelectrics, and frequency control, vol. 43, no. 6, pp. 1122-1129, 1996.

[12] J.-F. Aubry, M. Tanter, M. Pernot, J.-L. Thomas, and M. Fink, "Experimental demonstration of noninvasive transskull adaptive focusing based on prior computed tomography scans," The Journal of the Acoustical Society of America, vol. 113, no. 1, pp. 84-93, 2003.

[13] B. E. Treeby and B. Cox, "Modeling power law absorption and dispersion for acoustic propagation using the fractional laplacian," The Journal of the Acoustical Society of America, vol. 127, no. 5, pp. 2741-2748, 2010.

[14] K. Melde, A. G. Mark, T. Qiu, and P. Fischer, "Holograms for acoustics," Nature, vol. 537, no. 7621, pp. 518-522, 2016. 